Hidenori Ozaki • Yoko Watanabe • Keiko Ikeda Kiyoshi Kawakami

\title{
Impaired interactions between mouse Eya1 harboring mutations found in patients with branchio-oto-renal syndrome and Six, Dach, and G proteins
}

Received: November 1, 2001 / Accepted: December 4, 2001

\begin{abstract}
Mutations in the EYA1 gene are responsible for branchio-oto-renal (BOR) syndrome as well as for other ocular defects. Most of the mutations are located within or in the vicinity of the EYA domain, which is highly conserved in the EYA protein family. The EYA domain is required for protein-protein interactions, which are important to the biological function of EYA proteins. To determine how EYA1 mutations cause BOR syndrome and/or ocular defects, we tested the effects of Eya1 mutations on interactions with Six, Dach, and G proteins by mammalian two-hybrid and GST-pulldown assays. Defective interactions were noted between BOR-type mutations S486P and L504R of Eya1 and Dach1, G proteins, and some Six proteins. These mutations impaired the activation of transcription from a Six-responsive gene, myogenin, with Six5. S486P and L504R showed an altered digestion pattern with trypsin, and L504R also decreased the sensitivity to V8 protease digestion and produced a peptide fragment with a different $\mathrm{M}_{\mathrm{r}}$. Our results suggest that defective proteinprotein interactions of the mutations in the EYA domain underlie BOR syndrome and that SIX, DACH, and/or G proteins are possibly involved in the pathogenic processes.
\end{abstract}

Key words BOR syndrome - EYA1 - EYA domain . Six · Dach · G protein - Mammalian two-hybrid assay · GST-pulldown

\section{Introduction}

EYA family genes are homologues of Drosophila eyes absent (eya), which is required for compound eye formation. A clue to the molecular functions of $E Y A$ family gene products has been given by analyses using the Drosophila sys-

H. Ozaki · Y. Watanabe $\cdot$ K. Ikeda $\cdot$ K. Kawakami $(\bowtie)$ Department of Biology, Jichi Medical School, 3311-1 Yakushiji, Minamikawachi, Kawachi, Tochigi 329-0498, Japan

Tel. +81-285-58-7311; Fax +81-285-44-5476

e-mail: kkawakam@jichi.ac.jp tem. Loss-of-function mutations of eya, as well as those of sine oculis (so, one of Drosophila Six) and dachshund, result in a reduction or a complete loss of compound eye development in the fly (Bonini et al. 1993; Cheyette et al. 1994; Mardon et al. 1994), while ectopic expression of so or dachshund with eya synergistically induced compound eye formation (Pignoni et al. 1997; Chen et al. 1997). These genetic interactions were shown to be mediated through physical interactions between their gene products (Pignoni et al. 1997; Chen et al. 1997).

$E Y A 1$ was originally isolated as a gene responsible for branchio-oto-renal (BOR) syndrome, a human autosomal dominant disorder characterized by hearing impairment, branchial arch deformation, and variable severity of renal anomaly (Abdelhak et al. 1997b). In a recent report, EYA1 mutations were also found associated with certain ocular defects such as cataracts and iris anomaly (Azuma et al. 2000). To date, more than 20 mutations of $E Y A 1$ have been identified in patients with BOR syndrome or ocular defects, most of which are located in the EYA domain of the product (an amino acid substitution, a truncation, or a frameshift) (Abdelhak et al. 1997a, b; Azuma et al. 2000; Kumar et al. 1998).

The EYA domain is conserved among $E Y A$ family gene products and is required for protein-protein interaction. For example, in Drosophila, Eyes absent forms a complex with So and/or Dachshund through the Eya domain to induce synergistically compound eye formation (Pignoni et al. 1997; Chen et al. 1997), and mouse Eya forms a complex with Six, and synergistically activates the target gene promoter through nuclear translocation of Eya by Six, for which the Eya domain is indispensable (Ohto et al. 1999). These facts suggest that protein-protein interactions mediated by the EYA domain is important for functions of EYA proteins and for normal organogenesis.

To gain insight into the molecular basis of how EYA1 mutations cause BOR syndrome, we analyzed the effects of EYA1 mutations on such protein-protein interactions. For this purpose, we used the substitution and truncation mutations of EYA1 identified in patients with BOR syndrome (R275X, L472R, and R514G), ocular defects (E330K and 
S454P), or both (G393S) (Abdelhak et al. 1997b; Azuma et al. 2000) for the following reasons. (1) Amino acid residues at these mutations are conserved (E330K, G393S, and L472R) or homologous (R275X, S454P, and R514G) among $E Y A$ family genes, suggesting that the molecular functions of these residues are common to the $E Y A$ gene family. (2) In frame-shift mutations, sequences and lengths of subsequent amino acid stretches are not generally conserved, and these stretches might have unexpected gain-offunction. The observations that human EYA1 and mouse Eya1 are highly homologous $(99.6 \%$ identity in the EYA domain, $98.7 \%$ identity in the whole molecule) and that mice carrying mutations in Eyal manifest a BOR syndrome-like phenotype (Xu et al. 1999; Johnson et al. 1999) suggest that mouse Eyal functions in the same molecular context as human EYA1. Thus, we introduced the corresponding mutations found in human EYA1 into mouse Eya1 (R307X, S486P, and L504R for BOR type, E362K and $\mathrm{R} 546 \mathrm{G}$ for ocular type, and G425S for complex type) and performed molecular characterizations. We hypothesized that Eya1 mutations impair molecular interactions with cofactors mediated by the EYA domain and analyzed the interaction of these mutations with the known cofactors, Six, Dach, and G proteins.

We tested these interactions by mammalian two-hybrid assays and/or GST-pulldown assays. Furthermore, to examine whether the impaired interaction influences the transactivation function of Eya1 with Six, we performed reporter gene assays using a promoter of a Six-responsive gene, myogenin. Structural analyses of Eya1 mutations were also performed by protease digestion.

\section{Materials and methods}

Construction of plasmids

pHM6Eya1R307X, pHM6Eya1E362K, pHM6Eya1G425S, pHM6Eya1L504R, pHM6Eya1R546G, and pHM6Eya1S486P were constructed by introducing corresponding point mutations into pHM6Eya1 (Ohto et al. 1999) by cassette mutagenesis using the following sets of polymerase chain reaction (PCR) primers: 5'-CGTGGC CGAGGCTGAAGAAACAATA-3' and 5'-TATTGTTT CTTCA GCCTCGGCCACG-3' (R307X); 5'-GGACTAC GAATGAAAGAGATGATTT-3' and 5'-AAATCATCT CTTTCATTCGTAGTCC-3' (E362K); 5'-ACTGGTGTC CGA A GTGGTGTGGACT-3' and 5'-AGTCCACACCA CTTCGGACACCAGT-3' (G425S); 5'-CAAAGGTTTG GAGGGAAAGTGGTAT-3' and 5'-ATACCACTTTCC CTCCAAACCTTTG-3' (R546G); 5' -CTGAAGGCCCTC CCCCTCATCCACT-3' and 5'-AGTGGATGAGGGGG AGGGCCTTCAG-3' (S486P); and 5'-CAACTACGCAG CGCATCCCAGCATT-3' and 5'-AATGCTGGGATG GCTGCGTAGTTG-3' (L504R). The point mutations introduced are underlined.

pMEya1 was constructed as follows. The initiation codon (154)-HindIII (654) fragment was amplified by PCR so as to generate an EcoRI site at the 5'-terminus. The resulting EcoRI-HindIII fragment was inserted into the EcoRI/SalI sites of pM (Clontech Laboratories, Palo Alto, CA, USA) together with the HindIII (654)-XhoI (3'-terminus) fragment of pHM6Eya1.

pMEya1R307X was constructed by replacing the KpnI (661)-PstI (3'-terminus) fragment of pMEya1 with the KpnI (661)-Pst I (3'-terminus) fragment of pHM6Eya1R307X. pMEya1E362K, pMEya1G425S, pMEya1L504R, pMEya1R546G, and pMEya1S486P were constructed by replacing the BamHI fragment (1199 to 3'terminus) of pMEya1 with the BamHI fragment (1199 to 3 '-terminus) of pHM6Eya1E362K, pHM6Eya1G425S, pHM6Eya1L504R, pHM6Eya1R546G, and pHM6Eya1S486P, respectively.

pfSix1 was constructed as follows. Six 1 cDNA was cloned from a mouse mammary cancer cell SC-3 cDNA library, and the coding region was amplified by PCR using the following set of primers to generate a $K p n I$ site at the 5'-terminus and an $\mathrm{XbaI}$ site at the 3 '-terminus: 5'-GGGTA CCCATGTCGATGCTGCCGTCGT-3' and 5'-GCTCTA GATTAGGAACCCAAGTCCACCA-3'. The resulting PCR fragment was digested with $K p n I$ and $X b a \mathrm{I}$ and inserted into the $K p n \mathrm{I} / X b a \mathrm{I}$ sites of pFLAG-CMV-2 (Eastman Kodak, New Haven, CT, USA).

pVP16Six1, pVP16Six2, pVP16Six4, and pVP16Six5 were constructed as follows. The full length of the insert of pfSix1 was excised as the BamHI (blunt-ended)-XbaI fragment and inserted into the BamHI (blunt-ended)/XbaI sites of pVP16 (Clontech) (pVP16Six1); the full length of the insert of pfSix2 (Ohto et al. 1999) was excised as the BssHII (blunt-ended)-SmaI fragment and inserted into the SalI (blunt-ended) site of pVP16 (pVP16Six2); the full length of the insert of pfSix4 (Ohto et al. 1999) was excised as the $B g l I I$ (blunt-ended)-XbaI fragment and inserted into the BamHI (blunt-ended)/XbaI sites of pVP16 (pVP16Six4); the full length of the insert of pfSix 5 (Ohto et al. 1999) was excised as the $\mathrm{XbaI}$ (blunt-ended)-BglII (blunt-ended) fragment and inserted into the SalI (blunt-ended) site of pVP16 (pVP16Six5).

pfDach1 was constructed as follows. pBSmdac (kindly provided by $\mathrm{S}$. Krauss) was engineered to generate a HindIII site $5^{\prime}$ adjacent to the initiation codon (266). The HindIII-SspI (2520) fragment was cloned once into the HindIII/EcoRV sites of pBluescript KS+ (Stratagene, La Jolla, CA, USA). Then, the insert was excised as the HindIII-XbaI fragment and inserted into the HindIII/XbaI sites of pFLAG-CMV-2.

pVP16Gz and pVP16GzQ205L were constructed as follows. The NcoI (13)-XbaI (3'-terminus) fragments from pCMV5Gz and pCMV5GzQ205L (Itoh et al. 1986) were excised, blunt-ended, and inserted into the EcoRI (bluntended) site of pVP16. pVP16Gi2 and pVP16Gi2Q205L were constructed as follows. The 262-bp HaeIII fragments (34-295) from pCMV5Gi2 and pCMV5Gi2Q205L (Itoh et al. 1986) were inserted into the BamHI (blunt-ended) site of pVP16. EcoRI (5'-terminus)-BstXI (216) fragments of these plasmids and BstXI (216)-XbaI (3'-terminus) fragments of pCMV5Gi2 and pCMV5Gi2Q205L were 
simultaneously ligated to EcoRI/XbaI sites of pVP16, respectively.

pGEXSix1 was constructed as follows. The full length of the insert of pfSix 1 was excised as the EcoRV-XbaI (bluntended) fragment and inserted into the SmaI site of pGEX6P-1 (Amersham Pharmacia Biotech, Buckinghamshire, UK).

pGL3MG-1.7 was constructed by inserting the HindIII fragment (5' upstream 1.7-kb region of myogenin gene promoter) of pMGNLacZ (Fujisawa-Sehara et al. 1993) into the HindIII site of pGL3-Basic (Promega, Madison, WI, USA).

Cell culture and reporter gene assays

HEK 293 and NIH 3 T3 cells were grown in Dulbecco's modified Eagle's medium supplemented with 4.5 g glucose $/ 1$, $10 \%$ fetal bovine serum with 100 units $/ \mathrm{ml}$ penicillin, and $100 \mu \mathrm{g} / \mathrm{ml}$ streptomycin at $37^{\circ} \mathrm{C}$ under $5 \% \mathrm{CO}_{2}$. For mammalian two-hybrid assays, transfections into the HEK 293 cells were performed by the standard calcium phosphate method as described previously (Murakami et al. 1998) in 3.5-cm-diameter dishes. For coactivation activity of Eya1, transfections into the NIH $3 \mathrm{~T} 3$ cells were performed by using the SuperFect Transfection Reagent (QIAGEN, Hilden, Germany) in 24-well plates. Two days after the transfection, cells were collected and lysed for luciferase assays. All values were normalized to the internal controls of $\beta$-galactosidase activities.

GST-pulldown assays

GST, GST-Six2, GST-Six4, and GST-Six5 fusion proteins were prepared as described previously (Kawakami et al. 1996a, b). GST-Six1 fusion protein was prepared by using pGEXSix1 with the same procedure. pHM6Eya1 and its mutations were applied to the TNT Quick Coupled Transcription/Translation Systems (Promega, Madison, WI, USA) to obtain HA-tagged Eya1 and its mutation proteins labeled with $\left[{ }^{35}\right.$ S]methionine (Amersham Pharmacia Biotech).

GST, GST-Six1, GST-Six2, GST-Six4, and GST-Six5 fusion proteins bound to Glutathione Sepharose beads in the binding buffer $\left(50 \mathrm{mM} \mathrm{K}-\mathrm{PO}_{4}, \mathrm{pH} 7.5,150 \mathrm{mM} \mathrm{KCl}\right.$, $1 \mathrm{mM} \mathrm{MgCl} 2,10 \%$ glycerol, $1 \%$ Triton X-100) were incubated with ${ }^{35} \mathrm{~S}$-labeled HA-Eya1 and its mutations at $4{ }^{\circ} \mathrm{C}$ for $2 \mathrm{~h}$ with rotation. Supernatants were recovered as unbound fractions. Beads were washed five times with the binding buffer and dissolved in a sodium dodecyl sulfate (SDS) sample buffer, followed by sodium dodecyl sulfatepolyacrylamide gel electrophoresis (SDS-PAGE) and fluorography.

\section{Protease digestion}

${ }^{35}$ S-labeled Eya1 proteins and their mutations were incubated with V8 protease or trypsin (Sigma, St. Louis, MO, USA) in a digestion buffer [ $50 \mathrm{mM}$ Tris- $\mathrm{HCl}, \mathrm{pH} 7.6,25 \mathrm{mM}$
$\mathrm{NaCl}$, and $2 \mathrm{mM}$ ethylenediaminetetraacetate (EDTA)] at $4^{\circ} \mathrm{C}$ for $30 \mathrm{~min}$. After digestion, samples were added with SDS-sample buffer, heated at $95^{\circ} \mathrm{C}$ for $5 \mathrm{~min}$, and applied to SDS-PAGE, followed by fluorography.

\section{Results}

BOR-type mutations abolish the interaction between Dach1 and Eya1

Interactions between Dachshund and Eyes absent proteins in Drosophila were identified by yeast two-hybrid assays and GST-pulldown analyses (Chen et al. 1997). The interaction is mediated through the Eya domain and Dachshund domain 2 (DD2), both of which are conserved among various species (Chen et al. 1997; Xu et al. 1997; Caubit et al. 1999). We assessed the interaction between mouse Dach1 and mouse Eya1 by a mammalian two-hybrid assay. A plasmid expressing Eya1 or Eya1 mutations fused with the GAL4 DNA-binding domain (pMEya1 and its mutation derivatives) was used as bait (Table 1). FLAG-tagged Dach1-expressing plasmid, pfDach1, as prey, and a luciferase reporter plasmid consisting of five Gal4 DNAbinding sites upstream of the synthetic core promoter, pGLMRG5 (Ikeda et al. in press), were cotransfected into the HEK 293 cells. FLAG-tagged Dach1 was used because Dach1 itself shows a potent transactivation activity, and its activity is reduced in the context of the Dach1-VP16 fusion protein (K. Kawakami, unpublished observation). As shown in Fig. 1, pfDach1 stimulated the transcription of the

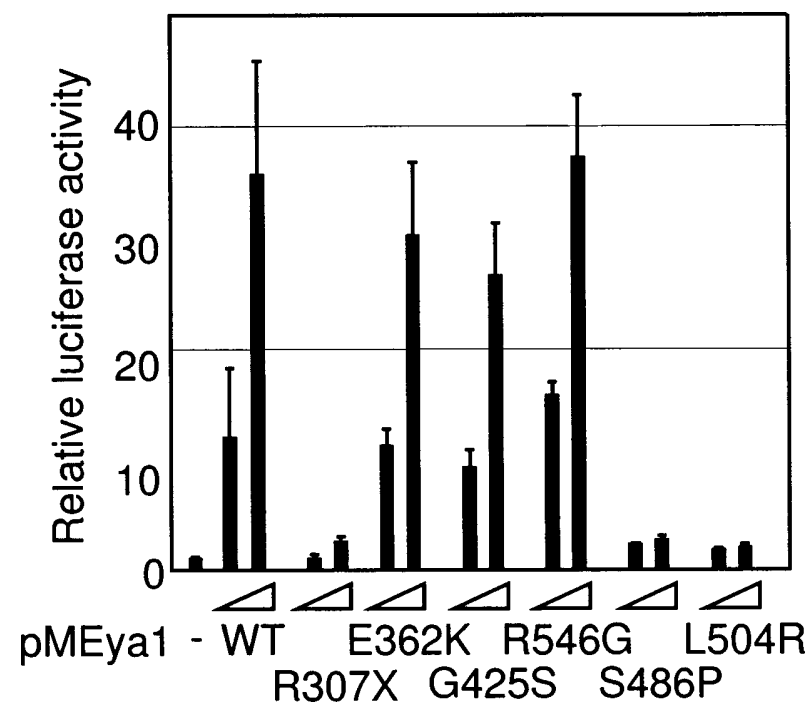

Fig. 1. Interaction between Eya1 and Dach1 in HEK 293 cells. Increasing amounts $(0.25$ or $0.50 \mu \mathrm{g})$ of pMEya1 wild type (WT) or the indicated Eya1 mutations were cotransfected with $0.3 \mu \mathrm{g}$ of pfDach1, and $0.5 \mu \mathrm{g}$ of pGL-MRG5 was cotransfected as a reporter gene. Luciferase activity in the cell lysate was normalized with $\beta$-galactosidase activity of pEFBOS $\beta$-gal as an internal control. The activity of each datum point is relative to that obtained by the control vector pM $(-)$. Each experiment was performed in triplicate, and the mean fold activation is shown with the standard deviation. Similar results were obtained from at least three independent experiments 
Table 1. Summary of the results of the mammalian two-hybrid assays analyzing the interaction of Eya1 mutations with the cofactors

\begin{tabular}{llllllll}
\hline $\begin{array}{l}\text { Human EYA1 } \\
\text { Mutations }\end{array}$ & wild type & E330K & R514G & G393S & $\begin{array}{l}\text { R275X } \\
\text { (truncation) }\end{array}$ & S454P & L472R \\
\hline $\begin{array}{llllll}\text { Phenotype } \\
\text { Corresponding }\end{array}$ & wild type & E362K & R546G & G425S & R307X & S486P & L504R \\
$\begin{array}{l}\text { mouse Eya1 } \\
\text { mutations }\end{array}$ & & & & & & & \\
Six1 & ++ & ++ & ++ & ++ & - & & \\
Six2 & ++ & ++ & ++ & ++ & - & ++ & - \\
Six4 & ++ & ++ & ++ & ++ & - & ++ & + \\
Six5 & ++ & ++ & ++ & ++ & - & ++ & + \\
Dach1 & ++ & ++ & ++ & ++ & - & - & - \\
Gaz Q205L & ++ & ++ & ++ & ++ & - & - & - \\
Gai2 Q205L & ++ & ++ & ++ & ++ & - & - & - \\
\hline
\end{tabular}

++ , strong interaction; + , weak interaction; - , undetectable interaction

reporter 12- to 36-fold in the presence of pMEya1 in a dosedependent manner, indicating that Dach1 interacts with the wild type Eya1.

We tested three BOR-type mutations, pMEya1R307X, pMEya1S486P, and pMEya1L504R, as well as the oculartype mutations, pMEya1E362K and pMEya1R546G, as bait. The former three BOR-type mutations marginally activated the transcription of the reporter gene $(<3$-fold), whereas the latter two ocular-type mutations enhanced the transcription (11- to 37-fold), similar to the wild type. Interestingly, a complex-type mutation pMEya1G425S, which has been described in a patient with both BOR syndrome and cataracts (Azuma et al. 2000), also enhanced the transcription (9- to 26-fold) similar to the wild type. These results indicate that the three BOR-type mutations abolished the interaction with Dach1, while the ocular- and complextype mutations retained the interaction with Dach1.

BOR-type mutations abolish the interaction between $\mathrm{G}$ proteins and Eya1

Human EYA2, another member of the $E Y A$ family of gene products, was found capable of binding to constitutively

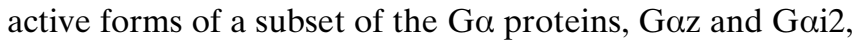
through the EYA domain, suggesting that EYA2 is one of the effector molecules of these G proteins (Fan et al. 2000). This prompted us to assess the interaction between Eya1 and these $\mathrm{G} \alpha$ proteins. In the following studies, we used the constitutive active mutations of rat GazQ205L and Gai2Q205L, which mimic the active form of each $\mathrm{G}$ protein (Fan et al. 2000). Mammalian two-hybrid assays were performed using pMEya1 as bait and plasmids expressing VP16-Ga fusion proteins, pVP16Gaz, pVP16GazQ205L, pVP16Gai2, or pVP16Gai2Q205L as prey (Fig. 2a). pVP16Gaz and pVP16Gai2 showed no activation of the reporter pGL-MRG5, whereas each constitutive active mutation, pVP16GazQ205L and pVP16Gai2Q205L, activated the transcription of the reporter from 33- to 79-fold and from 42- to 132-fold, respectively, in a dose-dependent manner. These results indicate that GazQ205L and Gai2Q205L interact with Eya1.
Next, we examined the effect of Eya1 mutations on the interaction between Eya1 and GazQ205L or Gai2Q205L (Figs. 2b, c). Three BOR-type mutations pMEya1R307X, pMEya1S486P, and pMEya1L504R showed no activation of the reporter. On the other hand, an ocular-type mutation, pMEya1E362K, enhanced the transcription of the reporter 52- to 103-fold (pVP16GazQ205L) or 69- to 116fold (pVP16Gai2Q205L). This enhancement was slightly higher than that of the wild-type pMEya1 (23- to 52-fold for pVP16GazQ205L and 62- to 89-fold for pVP16Gai2Q205L). The other ocular-type mutation pMEya1R546G enhanced 39- to 77-fold (pVP16GazQ205L) or 56- to 75-fold (pVP16Gai2Q205L), similar to wild-type pMEya1. The complex-type mutation pMEya1G425S showed a comparable level of activation (57- to 58-fold for pVP16GazQ205L and 57- to 96-fold for pVP16Gai2Q205L) of the reporter gene transcription to the pMEya1. The results indicate that three BOR-type mutations, R307X, S486P, and L504R, failed to interact with the constitutively active mutations of Gaz and Gai2, and that other Eya1 mutations retained their abilities to interact with these $\mathrm{G}$ proteins.

BOR-type mutations weaken the interaction between Six and Eya1

The functional synergy between Six2, Six4, or Six 5, and Eya1 has been reported with regard to transcription from their target gene (myogenin) promoter (Ohto et al. 1999). To examine whether the above Eya1 mutations influence the interaction with various Six, we performed mammalian two-hybrid assays (Fig. 3). A plasmid pVP16Six1, pVP16Six2, pVP16Six4, or pVP16Six5 expressing VP16Six1, -Six2, -Six4, or -Six5 fusion proteins, respectively, was used as prey. These Six proteins are expected to be involved in BOR syndrome on the basis of their expression in branchial arch, otic vesicle, and/or nephrogenic cord during embryogenesis. pMEya1 and its mutation derivatives were used as bait (Table 1). Various combinations of bait-prey were co-transfected into the HEK 293 cells with the reporter plasmid pGL-MRG5. 


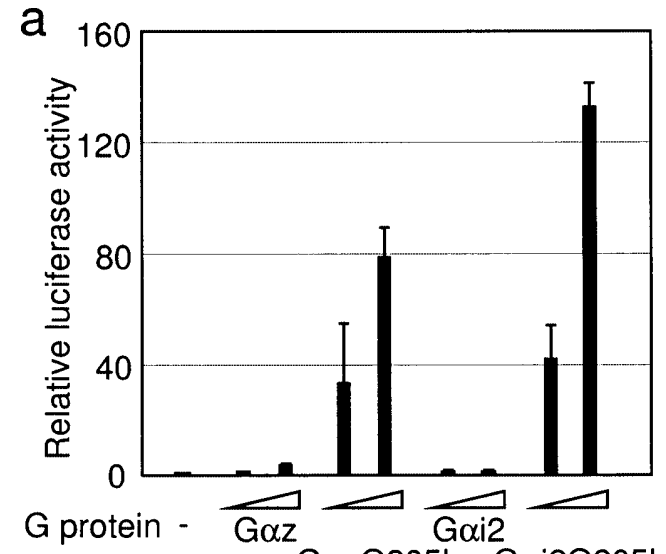

b
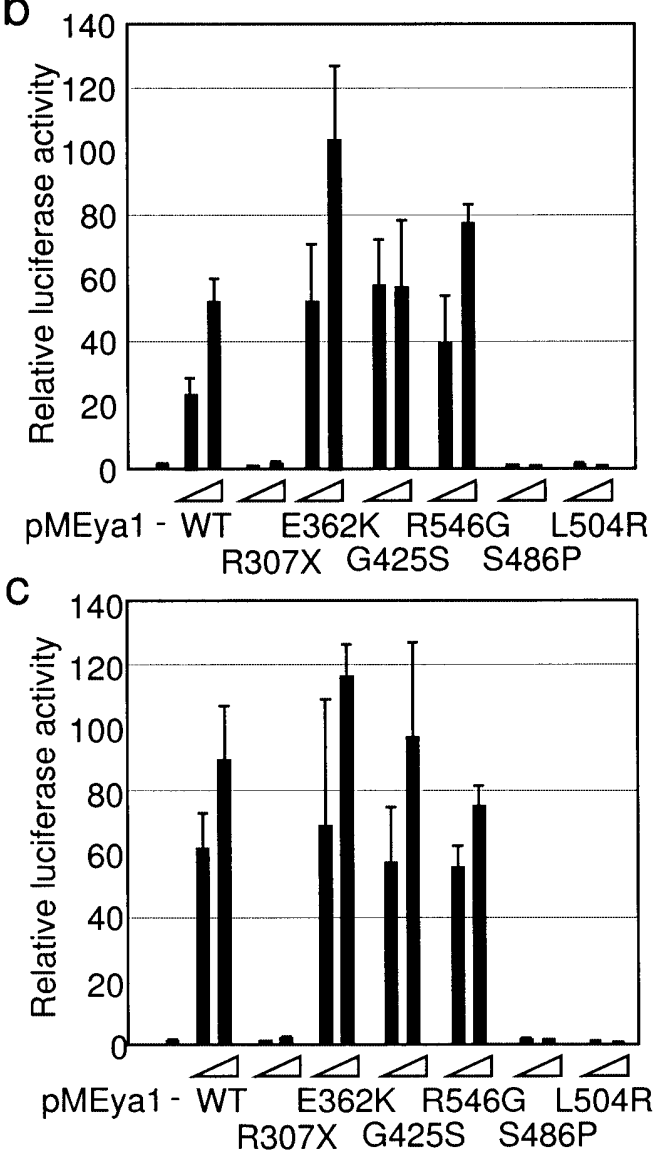

Fig. 2. Interaction between Eya1 and G proteins in HEK 293 cells. a Increasing amounts $(0.05$ and $0.1 \mu \mathrm{g})$ of pVP16G $\alpha z, \mathrm{pVP} 16 \mathrm{G} \alpha \mathrm{zQ} 205 \mathrm{~L}$, pVP16Gai2, or pVP16Gai2Q205L were cotransfected with $0.5 \mu \mathrm{g}$ of pMEya1. b, c Increasing amounts $(0.25$ and $0.5 \mu \mathrm{g})$ of pMEya1 (WT) or the indicated mutations were cotransfected with $0.05 \mu \mathrm{g}$ of

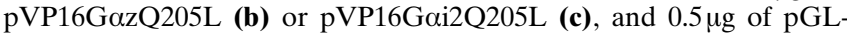
MRG5 was cotransfected as a reporter gene. Luciferase activity in the cell lysate was normalized with $\beta$-galactosidase activity of pEFBOS $\beta$ gal as an internal control. The activity of each datum point is relative to that obtained by the control vector $\mathrm{pM}(-)$. Each experiment was performed in triplicate, and the mean fold activation is shown with the standard deviation. Similar results were obtained from at least three independent experiments
For pVP16Six1, wild-type pMEya1 showed 14- to 15-fold activation of the reporter. pMEya1E362K (19- to 30-fold), pMEya1G425S (16- to 19-fold), pMEya1R546G (18- to 28fold), and pMEya1S486P (13- to 15-fold) showed comparable or even higher activation of the reporter, relative to pMEya1. In contrast, pMEya1R307X and pMEya1L504R showed little activation, up to 1 - and 3 -fold, respectively (Fig. 3a).

For pVP16Six2, wild-type pMEya1 showed 4.6- to 4.9fold activation of the reporter. pMEya1E362K (4.4- to 5.9fold), pMEya1G425S (5.3-fold), pMEya1R546G (4.8- to 5.6-fold), and pMEya1S486P (4.7- to 5.1-fold) showed comparable or a slightly higher activation of the reporter, relative to pMEya1. In contrast, pMEya1R307X showed little activation ( $<1.5$-fold $)$ and pMEya1L504R up to 2.3 - to 2.7-fold activation (Fig. 3b).

For pVP16Six4, wild-type pMEya1 showed 6.7- to 11fold activation of the reporter. pMEya1E362K (4.7- to 10fold), pMEya1G425S (5.2- to 10-fold), and pMEya1R546G (5.5- to 13-fold) showed comparable activation of the reporter, relative to pMEya1. In contrast, pMEya1R307X showed little activation (<1.1-fold) and S486P, and L504R showed 5.5- to 7.7-, and 2.3- to 3.3-fold activation, respectively (Fig. 3c).

In the case of pVP16Six 5 , wild-type pMEya1 showed 13- to 24-fold activation of the reporter. pMEya1E362K (13- to 35-fold), pMEya1G425S (22- to 30-fold), and pMEya1R546G (28- to 42-fold) showed comparable or higher activation of the reporter relative to pMEya1. In contrast, pMEya1R307X showed little activation $(<1.1$ fold), while S486P and L504R showed 10- to 11- and 3.2- to 4.2-fold activation, respectively (Fig. 3d).

These results indicate that the ocular-type and complextype mutations E362K, G425S, and R546G showed comparable or stronger interaction with any Six compared with wild-type Eya1, but that R307X abolishes the interaction with either Six. In contrast, S486P exhibits comparable or slightly weaker interaction with Six1, Six2, and Six4, but significantly weaker interaction with Six 5, and L504R shows marginal interactions with Six1 and Six5, although it retains significant interactions with Six2 and Six4 (summarized in Table 1).

To address whether the defects in interactions observed in mammalian two-hybrid assays are due to lack of the direct interaction between Eya1 and Six proteins, GSTpulldown assays were performed (Fig. 4). In vitro translated ${ }^{35}$ S-labeled Eya1 or its mutation proteins was incubated with Glutathione Sepharose beads bound to GST-Six fusion proteins. Bound and unbound fractions (denoted as B and S, respectively, in Fig. 4) were analyzed by SDS-PAGE, followed by fluorography. Wild-type Eya1 (WT) was observed in the bound fraction of any GST-Six fusion protein, but not in the bound fraction of GST alone, and it was observed in smaller quantity in the unbound fraction of any GST-Six fusion proteins than in the unbound fraction of GST alone. Similarly, E362K, G425S, and R546G were observed in larger quantities in the bound fraction of any GST-Six fusion protein than in the bound fraction of GST alone. In contrast, R307X, S486P, and L504R were mostly 
Fig. 3. Interaction between Eya1 mutations and Six proteins in HEK 293 cells; $0.5 \mu \mathrm{g}$ of pVP16Six1 (a), pVP16Six2 (b), pVP16Six4 (c) or pVP16Six5 (d) was cotransfected with increasing amounts $(0.25$ or $0.5 \mu \mathrm{g})$ of pMEya1 (WT) or the indicated mutations, and $0.5 \mu \mathrm{g}$ of pGLMRG5 was cotransfected as a reporter gene. Luciferase activity in the cell lysate was normalized with $\beta$-galactosidase activity of pEFBOS $\beta$-gal as an internal control. The activity of each datum point is relative to that obtained by the control vector $\mathrm{pM}$ $(-)$. Each experiment was performed in triplicate, and the mean fold activation is shown with the standard deviation. Similar results were obtained from at least two independent experiments
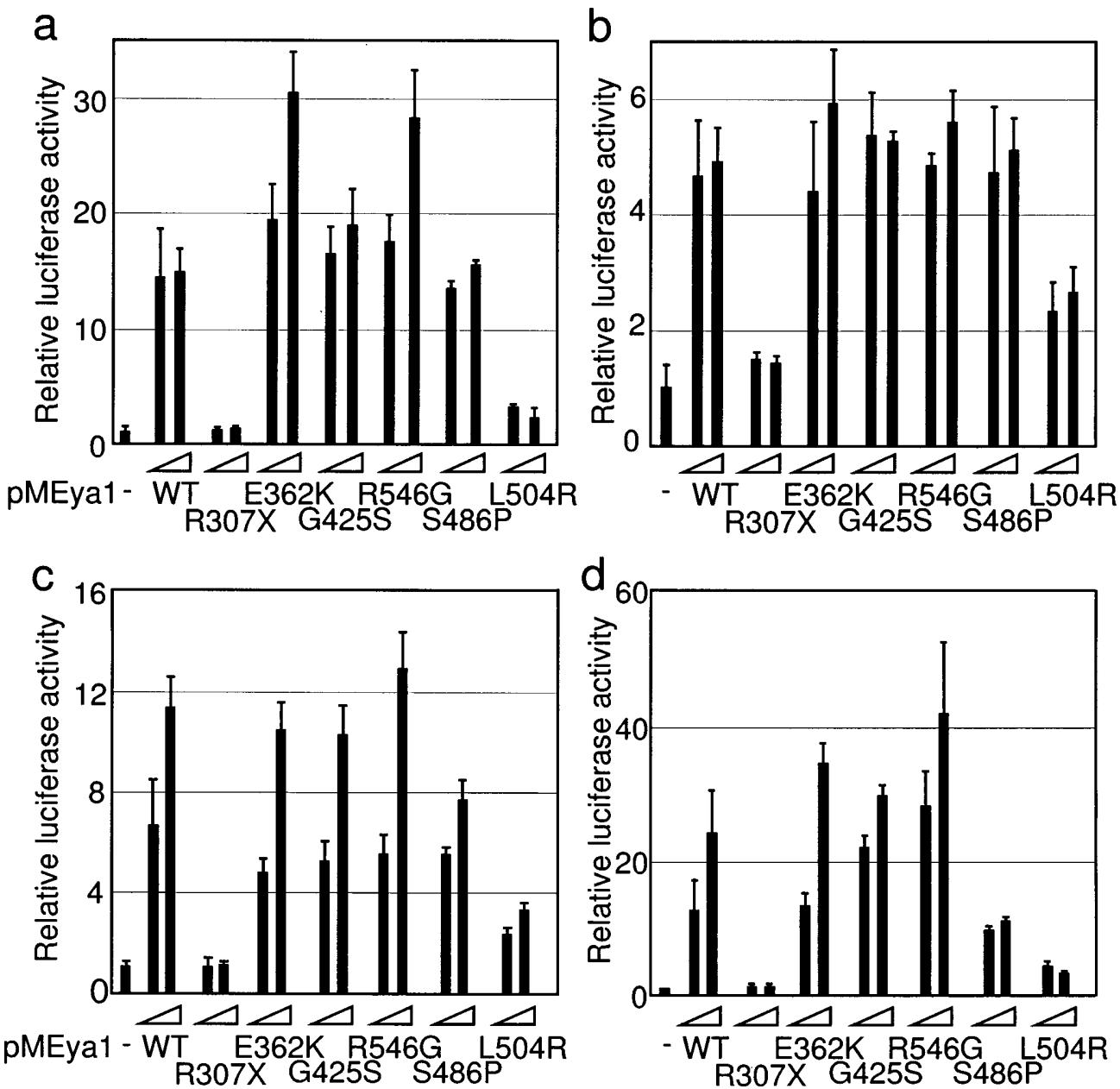

observed in the unbound fraction of all GST-Six proteins and GST alone, and little or none of these proteins were observed in the bound fraction of any GST-Six. These results indicate that the wild-type Eya1 efficiently binds to all of the GST-Six fusion proteins, and that E362K, G425S, and R546G interact with any GST-Six fusion proteins at significant, but varying levels. In contrast, BOR-type mutations, R307X, S486P, and L504R, did not show significant binding to any GST-Six proteins.

BOR-type mutations perturb the transcription from the myogenin promoter

As a coactivator, Eya1 synergistically activates myogenin gene transcription with Six2, Six4, and Six5, which bind to its promoter region (Ohto et al. 1999). We checked whether mutated versions of Eya1 could activate the myogenin gene transcription with Six5 as well as wild-type Eya1. Wild-type pHM6Eya1 activated transcription from the myogenin promoter with Six5 about 4.6-fold. In contrast, pHM6Eya1S486P and pHM6Eya1L504R showed little or no activation: 1.0- and 0.6-fold, respectively. When we used mutations of pHM6Eya1E362K and pHM6Eya1G425S, comparable levels of activation (5.4- and 5.0-fold, respectively) were observed. pHM6Eya1R546G showed a slightly lower level of activation, around 3.2-fold (Fig. 5). These transactivations of the myogenin promoter by wild-type Eya1 and mutations of Eya1 exhibited a good correlation with the two-hybrid interactions depicted in Fig. 3. This indicates that the defective coactivator function of Eya1 could be attributed to the reduced physical interactions between Eya1 and Six in transactivation of the myogenin promoter.

BOR-type mutations alter sensitivity to V8 protease and trypsin digestion

The fact that two BOR-type substitution mutations, S486P and L504R, simultaneously lost the interaction with Dach1 and $G$ proteins, strongly suggests a gross conformational change in these mutations. To address this possibility, we performed V8 protease mapping. In the case of S486P, we did not observe any difference in sensitivity or in the digestion pattern compared with those of the wild type (Fig. 6a, lanes 1-6 and 7-12). In contrast, we detected an alteration of the digestion pattern by V8 protease in L504R (Fig. 6a, lanes 13-18). Two- to 3-fold higher amounts of the undigested polypeptide (denoted as A) remained in L504R than in the wild type when we added $200 \mathrm{ng}$ to $2 \mu \mathrm{g}$ of V8 protease (compare lanes 16-18 with lanes 4-6), while the pat- 


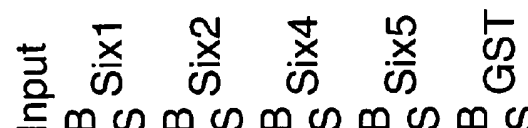

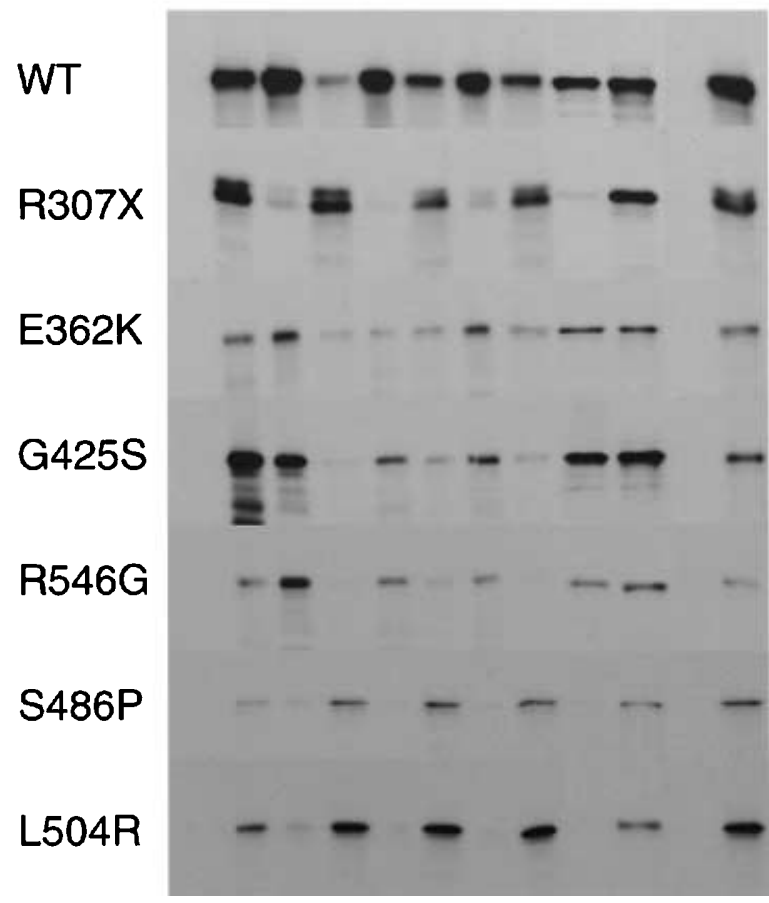

Fig. 4. Direct interaction between Eya1 and Six proteins in vitro. One microgram of GST-Six $1,0.5 \mu \mathrm{g}$ of GST-Six2, $1 \mu \mathrm{g}$ of GST-Six4, or $5 \mu \mathrm{g}$ of GST-Six 5 fusion protein bound to Glutathione Sepharose beads was incubated with in vitro translated, ${ }^{35} \mathrm{~S}$-labeled Eya1 (WT) or the indicated mutations at $4{ }^{\circ} \mathrm{C}$ for $2 \mathrm{~h}$. Ten percent of input, $50 \%$ of bound proteins $(B)$ and $17 \%$ of supernatant $(S)$ were electrophoresed on a $12 \%$ polyacrylamide sodium dodecyl sulfate gel followed by fluorography

terns of fragments $\mathrm{D}, \mathrm{E}$, and $\mathrm{F}$ in L504R digestion did not change relative to the wild-type digestion (compare lanes 15-18 with lanes 3-6). Fragment $\mathrm{C}$ was detected at higher levels in L504R than in the wild type with the addition of $650 \mathrm{ng}$ and $2 \mu \mathrm{g}$ of V8 protease (lanes 17 and 18, compare with lanes 5 and 6 ). Furthermore, fragment B was hardly detected, and fragments with slightly different mobility from that of fragment B appeared (Fig. 6a, lanes 14-18). In addition, fragment B' was present at a higher level with $2 \mu \mathrm{g}$ of V8 protease in L504R compared with the wild type (discerned by short exposure, data not shown). These results indicate that L504R is more resistant to V8 protease digestion, and the digestion pattern is distinct from those of the wild type and S486P, suggesting a different conformation of L504R.

We also performed protease mapping using trypsin and detected alterations of the digestion pattern in S486P and L504R (Fig. 6b). Far more undigested polypeptide (denoted as A) remained in both mutations than in the wild type when we added $12 \mathrm{ng}$ of trypsin (compare lanes 11 and 17 with lane 5). Fragment B was hardly detected at $4 \mathrm{ng}$ of trypsin (Fig. 6b, lanes 10 and 16). Fragments D, E, and H were missing or detected only at lower levels in these muta-

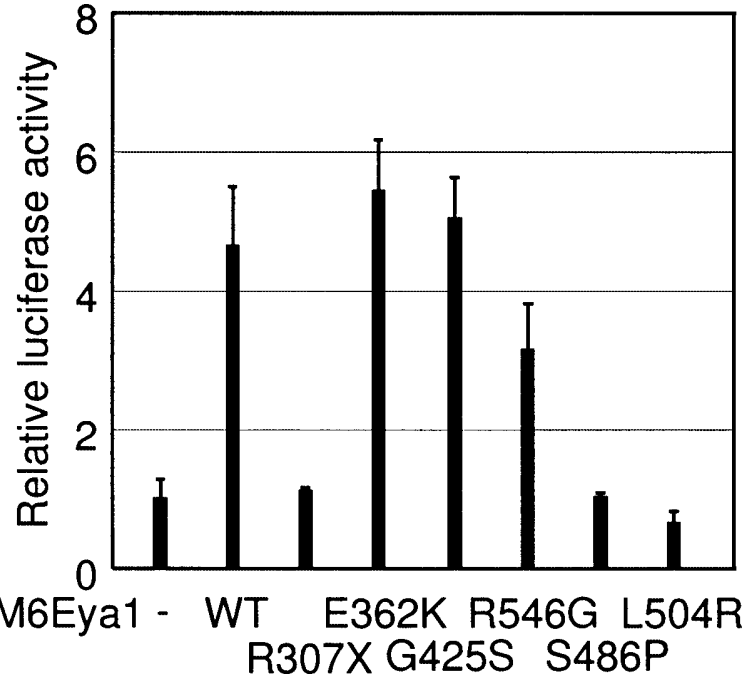

Fig. 5. Effects of Eya1 mutations on myogenin promoter transcription in NIH 3 T3 cells. One hundred nanograms of pfSix 5 was cotrancfected with $300 \mathrm{ng}$ of pHM6Eya1 or its mutations. Two micrograms of pGL3MG-1.7 was cotransfected as a reporter gene. Luciferase activity in the cell lysate was normalized with $\beta$-galactosidase activity of pEFBOS $\beta$-gal as an internal control. The activity of each datum point is relative to that obtained by the control vector pHM6 (-). Each experiment was performed in triplicate, and the mean fold induction is shown with the standard deviation. Similar results were obtained from at least two independent experiments

tions than in the wild type with the addition of $40 \mathrm{ng}$ of trypsin, while fragment I was detected at higher levels (lanes 12 and 18, compare with lane 6). The patterns of the other proteolytic fragments, C, F, G, and I in S486P and L504R digestion were unchanged relative to those in the wild type digestion (Fig. 6b, compare lanes 12 and 18 with lane 6). These results indicate that S486P and L504R are more resistant to trypsin digestion and that the digestion patterns of these mutations are distinct from that of the wild type, suggesting an altered conformation of S486P and L504R.

\section{Discussion}

In this study, we found that two BOR-type Eya1 substitution mutations, S486P and L504R, and a truncation mutation, $\mathrm{R} 307 \mathrm{X}$, were defective in protein-protein interactions mediated by the Eya domain. Our results also suggest the possible involvement of Six, Dach1, and G proteins in the pathogenicity of BOR syndrome.

The S486P and L504R mutations are located in the central region of the Eya domain, corresponding to the subregion of the Eya domain of Drosophila Eyes absent (EF2), which is required for the association with Drosophila Dachshund (Bui et al. 2000). Dachshund and Eyes absent form a complex, and synergistically induce compound eye formation in Drosophila (Chen et al. 1997). Similarly in chicken, Dach2 and Eya2 physically interact and synergistically induce myogenic gene expression (Heanue et al. 1999). 


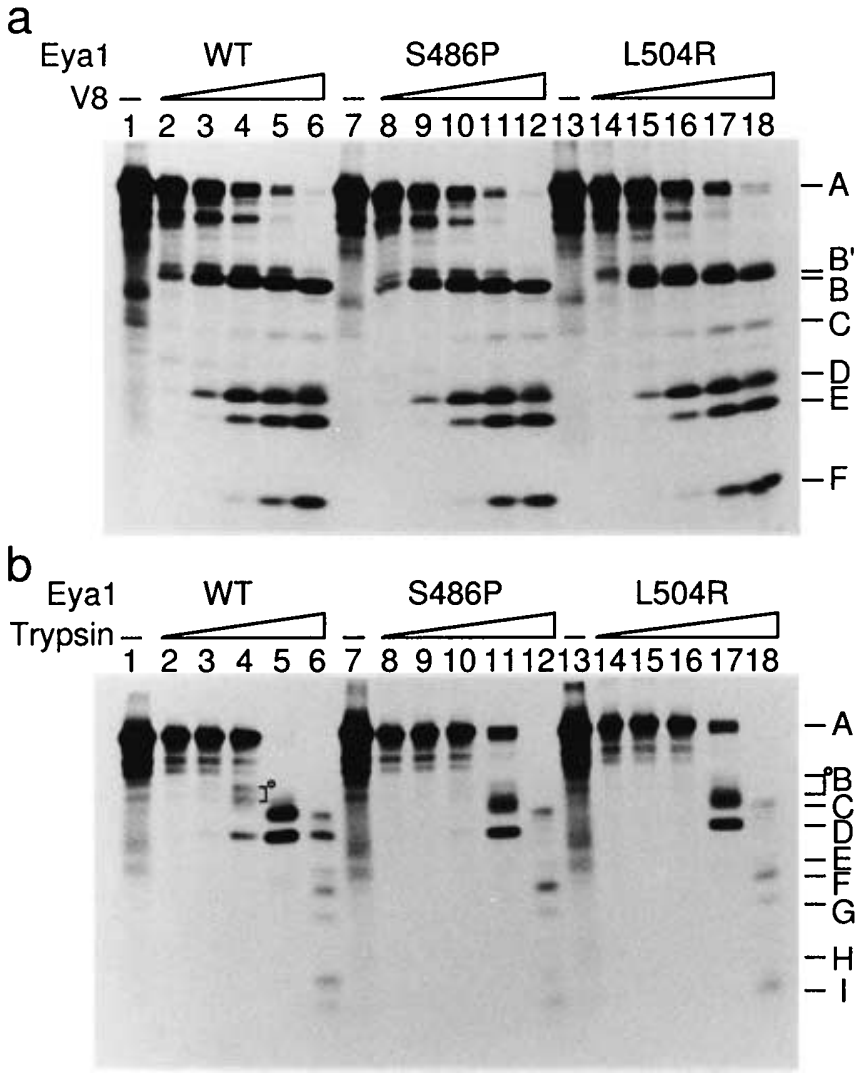

Fig. 6. Protease digestion profiles of Eya1 wild type and its mutations by V8 protease (a) and trypsin (b). ${ }^{35}$ S-labeled Eya1 wild type or its mutations translated in vitro in rabbit reticulocyte lysate was digested without (panel a, lanes 1, 7, and 13) or with $20 \mathrm{ng}$ (panel a, lanes 2, 8, and 14), $65 \mathrm{ng}$ (panel a, lanes 3, 9, and 15), $200 \mathrm{ng}$ (panel a, lanes 4, 10, and 16), 650 ng (panel a, lanes 5, 11, and 17), and $2 \mu \mathrm{g}$ (panel a, lanes 6, 12 , and 18) of V8 protease or without (panel b, lanes 1, 7, and 13) or with $0.4 \mathrm{ng}$ (panel b, lanes 2, 8, and 14), 1.2ng (panel b, lanes 3, 9, and 15), $4 \mathrm{ng}$ (panel b, lanes 4, 10, and 16), 12 ng (panel b, lanes 5, 11, and 17), and $40 \mathrm{ng}$ (panel b, lanes 6,12 , and 18 ) of trypsin for $30 \mathrm{~min}$ at $4^{\circ} \mathrm{C}$ and analyzed by sodium dodecyl sulfate-polyacrylamide gel electrophoresis followed by fluorography. Undigested full-length polypeptides are denoted as $A$. The major proteolytic fragments are designated $B$ to $F$ (panel $a$ ) and $B$ to $H$ (panel $b$ ), according to their electrophoretic mobilities

Thus, it is possible that the interaction between DACH1 and EYA1 is necessary for the development of certain organs. Actually, both Eya1 and Dach1 are expressed in otic vesicles and branchial arches in mouse (Xu et al. 1997; Caubit et al. 1999). The abrogation of such interaction by these BOR-type mutations may perturb the normal organogenesis, leading to BOR syndrome.

We also found that Eya1 interacted with the active form of the two types of $\mathrm{G}$ proteins, Gaz and Gai2 (Fig. 2a). These $\mathrm{G}$ proteins have been reported to bind to EYA2 and negatively regulate its coactivator function by inhibiting the nuclear translocation of EYA 2 mediated by Six1 and Six4 (Fan et al. 2000). We propose that the $G$ proteins also modulate the distribution of Eya1 proteins and regulate the Eya1 functions in organogenesis, and that impaired interaction of Eya1 mutations with these $\mathrm{G}$ proteins may result in their aberrant subcellular distribution, which might be involved in the onset of BOR syndrome.

Mostly consistent results were noted in the mammalian two-hybrid and GST-pulldown assays for the interaction of Eya1 mutations with four types of Six proteins (Figs. 3 and 4). The BOR-type truncation mutation, R307X, and the substitution mutations, S486P and L504R, showed little interaction with Six1, Six2, Six4, or Six 5 by GST-pulldown assays (Fig. 4). R307X showed little or no interaction with any Six protein by mammalian two-hybrid assays, whereas L504R showed weak but significant two-hybrid interaction with any Six, and S486P reduced two-hybrid interactions only with Six 4 and Six 5 (Fig. 3). The difference between the results of the two assays may be due to their different sensitivities or may be explained by the involvement of a third factor in the living cells, which bridges or stabilizes the interaction between Eya1 and the Six proteins. Impaired interaction of Six 4 and Six 5 with all three BOR-type Eya1 mutations suggests the involvement of these Six proteins in the pathogenicity of BOR syndrome. In fact, Six 4 as well as Six1 are expressed in branchial arches, otic vesicles, and nephrogenic tissues (Oliver et al. 1995; Ohto et al. 1998; Ozaki et al. 2001), and Six5 as well as Six 2 are expressed in branchial arches and nephrogenic tissues (Oliver et al. 1995; Ohto et al. 1998; Klesert et al. 2000). Thus, it is possible that BOR-type mutations reduce the interaction with these Six proteins to perturb the expression of the Six-responsive genes, leading to BOR syndrome.

In contrast to the BOR-type mutations, the ocular- and complex-type mutations E362K, G425S, and R546G did not show any defects in two-hybrid, GST-pulldown, and transactivation assays. This finding suggests that unidentified factors other than Six, Dach, or G proteins are probably involved in cascades that lead to ocular defects. Alternatively, a possible impairment of transactivation of these Eya1 mutations might not be the main pathway to ocular defects. However, the fact that Six5-deficient mice developed cataracts (Klesert et al. 2000; Sarkar et al. 2000) suggests the involvement of Six 5 in ocular developmental defects. Furthermore, Six 5 and Eya1 showed high synergistic activation (Fig. 5). In this context, it is plausible that defects of transactivation of these mutations of Eya1 would be detected if we used the promoter of the relevant target genes of Six5, which operates in the lens.

The BOR-type substitution mutations, S486P and L504R, were associated with the disappearance or weakening of the interaction with structurally unrelated groups of proteins (Figs. 1-4). In Drosophila Eyes absent, the regions that interact with So and Dachshund are reported to be distinct (EF1 and EF2 subdomains of the Eya domain, respectively) (Bui et al. 2000). S486P and L504R are located within the region corresponding to EF2, not to EF1. Nevertheless, these two mutations were not only associated with the loss of interaction with Dach1, but also with the weakening of the interaction with Six5. Considering that the serine to proline substitution in S486P changes the structure of the backbone and that the leucine to arginine substitution in L504R changes the charge and the size of the amino acid side chains, these results suggest a gross conforma- 
tional change in these mutations. In fact, S486P and L504R showed a low sensitivity to and a distinct pattern of trypsin digestion compared with those of wild-type Eya1 (Fig. 6b). L504R also showed a low sensitivity to and a distinct pattern of V8 protease digestion (Fig. 6a). These results suggest that the S486P and L504R mutations cause the conformational changes that disturb the protein-protein interactions mediated by the EYA domain.

In contrast to our expectation, the complex-type mutation G425S, which was identified in a patient with combined BOR syndrome and ocular defects (Azuma et al. 2000), interacted with Dach, the two G proteins, and the four Six proteins and held the transactivation activity to levels similar to those in the wild-type Eya1. Patients carrying this mutation may need further evaluation, including a search for additional mutations outside the exons of the EYAl loci, for example in the promoter region. However, the possibility is not excluded that the G425S mutation, as well as S486P and L504R, may disturb the interaction of Eya1 with unidentified cofactor(s) essential for normal organogenesis.

Structural studies including determination of the threedimensional structure of EYA1, combined with biochemical analyses including the search for other factors binding to EYA1 and the target genes of SIX, should further enhance our understanding of the function of EYA1 and the pathogenic mechanisms of BOR syndrome.

Acknowledgments We thank $\mathrm{H}$. Itoh for providing pCMVGz, pCMVGzQ205L, pCMV5Gi2, and pCMVQ205L, S. Krauss for providing pBSmdac, and A. Tanaka for providing the SC-3 library. We also thank S. Sato for the construction of pfDach1, cloning of mouse Six1, and helpful discussion, and $\mathrm{H}$. Ozaki-Ohto for the construction of pMEya1, pfSix1, and pGL3MG-1.7. This work was supported by grants from the Ministry of Education, Culture, Sports, Science and Technology of Japan, from the the Ministry of Health, Labor and Welfare of Japan, from the Sankyo Foundation of Life Science, and from the Cell Science Research Foundation.

\section{References}

Abdelhak S, Kalatzis V, Heilig R, Compain S, Samson D, Vincent C, Levi-Acobas F, Cruaud C, Le Merrer M, Mathieu M, König R, Vigneron J, Weissenbach J, Petit C, Weil D (1997a) Clustering of mutations responsible for branchio-oto-renal (BOR) syndrome in the eyes absent homologous region (eyaHR) of EYA1. Hum Mol Genet 6:2247-2255

Abdelhak S, Kalatzis V, Heilig R, Compain S, Samson D, Vincent C, Weil D, Cruaud C, Sahly I, Leibovici M, Bitner-Glindzicz M, Francis M, Lacombe D, Vigneron J, Charachon R, Boven K, Bedbeder P, Van Regemorter N, Weissenbach J, Petit C (1997b) A human homologue of the Drosophila eyes absent gene underlies branchiooto-renal (BOR) syndrome and identifies a novel gene family. Nat Genet 15:157-164

Azuma N, Hirakiyama A, Inoue T, Asaka A, Yamada M (2000) Mutations of a human homologue of the Drosophila eyes absent gene (EYA1) detected in patients with congenital cataracts and ocular anterior segment anomalies. Hum Mol Genet 9:363-366

Bonini NM, Leiserson WM, Benzer S (1993) The eyes absent gene: genetic control of cell survival and differentiation in the developing Drosophila eye. Cell 72:379-395

Bui QT, Zimmerman JE, Liu H, Bonini NM (2000) Molecular analysis of Drosophila eyes absent mutants reveals features of the conserved Eya domain. Genetics 155:709-720
Caubit X, Thangarajah R, Theil T, Wirth J, Nothwang HG, Rüther U, Krauss S (1999) Mouse Dac, a novel nuclear factor with homology to Drosophila dachshund shows a dynamic expression in the neural crest, the eye, the neocortex, and the limb bud. Dev Dyn 214:6680

Chen R, Amoui M, Zhang Z, Mardon G (1997) Dachshund and eyes absent proteins form a complex and function synergistically to induce ectopic eye development in Drosophila. Cell 91:893-903

Cheyette BNR, Green PJ, Martin K, Garren H, Hartenstein V, Zipursky SL (1994) The Drosophila sine oculis locus encodes a homeodomain-containing protein required for the development of the entire visual system. Neuron 12:977-996

Fan X, Brass LF, Poncz M, Spitz F, Maire P, Manning DR (2000) The $\alpha$ subunits of $\mathrm{Gz}$ and $\mathrm{Gi}$ interact with the eyes absent transcription cofactor Eya2, preventing its interaction with the six class of homeodomain-containing proteins. J Biol Chem 275:3212932134

Fujisawa-Sehara A, Hanaoka K, Hayasaka M, Hiromasa-Yagami T, Nabeshima Y (1993) Upstream region of the myogenin gene confers transcriptional activation in muscle cell lineages during mouse embryogenesis. Biochem Biophys Res Commun 191:351-356

Heanue TA, Reshef R, Davis RJ, Mardon G, Oliver G, Tomarev S, Lassar AB, Tabin CJ (1999) Synergistic regulation of vertebrate muscle development by Dach2, Eya2, and Six1, homologs of genes required for Drosophila eye formation. Genes Dev 13:32313243

Ikeda K, Stuehler T, Meisterernst M (2001) The H1 and H2 regions of the activation domain of herpes simplex virion protein 16 stimulate transcription through distinct molecular mechanisms. Genes Cells (in press)

Itoh H, Kozasa T, Nagata S, Nakamura S, Katada T, Ui M, Iwai S, Ohtsuka E, Kawasaki H, Suzuki K, Kaziro Y (1986) Molecular cloning and sequence determination of cDNAs for $\alpha$ subunits of the guanine nucleotide-binding proteins $\mathrm{Gs}, \mathrm{Gi}$, and $\mathrm{Go}$ from rat brain. Proc Natl Acad Sci U S A 83:3776-3780

Johnson KR, Cook SA, Erway LC, Matthews AN, Sanford LP, Paradies NE, Friedman RA (1999) Inner ear and kidney anomalies caused by IAP insertion in an intron of the Eyal gene in a mouse model of BOR syndrome. Hum Mol Genet 8:645-653

Kawakami K, Ohto H, Ikeda K, Roeder RG (1996a) Structure, function and expression of a murine homeobox protein AREC3, a homologue of Drosophila sine oculis gene product, and implication in development. Nucleic Acids Res 24:303-310

Kawakami K, Ohto H, Takizawa T, Saito T (1996b) Identification and expression of six family genes in mouse retina. FEBS Lett 393:259263

Klesert TR, Cho DH, Clark JI, Maylie J, Adelman J, Snider L, Yuen EC, Soriano P, Tapscott SJ (2000) Mice deficient in Six5 develop cataracts: implications for myotonic dystrophy. Nat Genet 25:105109

Kumar S, Deffenbacher K, Cremers CW, Van Camp G, Kimberling WJ (1998) Branchio-oto-renal syndrome: identification of novel mutations, molecular characterization, mutation distribution, and prospects for genetic testing. Genet Test 1:243-251

Mardon G, Solomon NM, Rubin GM (1994) dachshund encodes a nuclear protein required for normal eye and leg development in Drosophila. Development 120:3473-3486

Murakami Y, Ohto H, Ikeda U, Shimada K, Momoi T, Kawakami K (1998) Promoter of $m D M A H P /$ Six 5: differential utilization of multiple transcription initiation sites and positive/negative regulatory elements. Hum Mol Genet 7:2103-2112

Ohto H, Takizawa T, Saito T, Kobayashi M, Ikeda K, Kawakami K (1998) Tissue and developmental distribution of Six family gene products. Int J Dev Biol 42:141-148

Ohto H, Kamada S, Tago K, Tominaga SI, Ozaki H, Sato S, Kawakami K (1999) Cooperation of Six and Eya in activation of their target genes through nuclear translocation of Eya. Mol Cell Biol 19:68156824

Oliver G, Wehr R, Jenkins NA, Copeland NG, Cheyette BNR, Hartenstein V, Zipursky SL, Gruss P (1995) Homeobox genes and connective tissue patterning. Development 121:693-705

Ozaki H, Watanabe Y, Takahashi K, Kitamura K, Tanaka A, Urase K, Momoi T, Sudo K, Sakagami J, Asano M, Iwakura Y, Kawakami K (2001) Six 4, a putative myogenin gene regulator, is not essential for mouse embryonal development. Mol Cell Biol 21:3343-3350 
Pignoni F, Hu B, Zavitz KH, Xiao J, Garrity PA, Zipursky SL (1997) The eye-specification proteins So and Eya form a complex and regulate multiple steps in Drosophila eye development. Cell 91:881891

Sarkar PS, Appukuttan B, Han J, Ito Y, Ai C, Tsai W, Chai Y, Stout JT, Reddy S (2000) Heterozygous loss of Six 5 in mice is sufficient to cause ocular cataracts. Nat Genet 25:110-114
Xu PX, Woo I, Her H, Beier DR, Maas RL (1997) Mouse Eya homologues of the Drosophila eyes absent gene require Pax6 for expression in lens and nasal placode. Development 124:219231

Xu PX, Adams J, Peters H, Brown MC, Heaney S, Maas R (1999) Eyal-deficient mice lack ears and kidneys and show abnormal apoptosis of organ primordia. Nat Genet 23:113-117 